\title{
Prevalence and Correlates of Asymptomatic Malaria and Anemia on First Antenatal Care Visit among Pregnant Women in Southeast, Tanzania
}

\author{
Eulambius M. Mlugu ${ }^{1,2}$, Omary Minzi ${ }^{3}$, Appolinary A. R. Kamuhabwa ${ }^{3}$ and Eleni Aklillu $1, *$ (D) \\ 1 Division of Clinical Pharmacology, Department of Laboratory Medicine, Karolinska Institutet at Karolinska, \\ University Hospital, Huddinge, 14186 Stockholm, Sweden; mlugusonlove@gmail.com \\ 2 Department of Pharmaceutics, School of Pharmacy, Muhimbili University of Health and Allied Sciences, \\ Dar es Salaam 0702172, Tanzania \\ 3 Department of Clinical Pharmacy and Pharmacology, School of Pharmacy, Muhimbili University of Health \\ and Allied Sciences, Dar es Salaam 0702172, Tanzania; minziobejayesu@gmail.com (O.M.); \\ enali2012@gmail.com (A.A.R.K.) \\ * Correspondence: eleni.aklillu@ki.se
}

Received: 7 April 2020; Accepted: 28 April 2020; Published: 30 April 2020

\begin{abstract}
Asymptomatic malaria and anemia during pregnancy increase the risk of negative birth outcomes. This cross-sectional study investigated the prevalence and correlates of asymptomatic malaria and anemia during first antenatal care (ANC) visit among pregnant women in a rural district, Tanzania. HIV-uninfected pregnant women without symptoms of malaria $(n=819)$ attending their first ANC at Kibiti Health Centre were enrolled from February 2017 to February 2018. Asymptomatic malaria was detected by malaria rapid-diagnostic tests (mRDT) and real-time PCR. Hemoglobin concentration was determined by HemoCue Hemoglobin 201+. The study outcomes were the prevalence of asymptomatic malaria and anemia (Hemoglobin level $<11 \mathrm{~g} / \mathrm{dL}$ ). The overall prevalence of asymptomatic malaria was $36.4 \%$ (95\% CI: 33.1, 39.8). The monthly prevalence of asymptomatic malaria remained $>25 \%$ throughout the year, and the highest prevalence $(40 \%)$ was recorded during the rainy season. Asymptomatic malaria was significantly associated with primigravida, younger maternal age, and anemia. The prevalence of anemia was 68.5\% (95\% CI: 65.2, 71.6). Asymptomatic malaria, primigravida, younger maternal age and low Body Mass Index were significant predictors of low hemoglobin concentration. We report high prevalence of asymptomatic malaria and anemia among pregnant women on the first ANC visit. Screening of malaria and anemia during the first ANC visit is recommended for targeted interventions.
\end{abstract}

Keywords: pregnancy; pregnant women; malaria; asymptomatic malaria; anemia; antenatal care

\section{Introduction}

Malaria and anaemia in pregnancy are significant maternal health problems causing an increased risk for maternal death, stillbirth, spontaneous abortion, and low birth weight [1,2]. The World Health Organization (WHO) recommends antenatal care (ANC) involving intermittent preventive treatment in pregnancy with sulfadoxine-pyrimethamine (IPTp-SP) and folic acid supplementation for prevention of malaria and anemia respectively in all pregnant women living in endemic countries. However, in most cases, pregnant women begin attending ANC after the first trimester has passed, and therefore, do not fully benefit from the service [3,4]. Thus, evaluating the efficiency of existing interventions for prevention of malaria and anemia provides valuable information for better planning and implementation of public health programs. 
In low-malaria-transmission areas, there is poor acquired immunity; hence, severe symptomatic episodes of malaria in pregnancy are common, requiring prompt diagnosis and treatment [5]. On the other hand, due to acquired immunity in moderate and high malaria transmission areas, malaria is associated with asymptomatic cases. Asymptomatic infections present no clinical manifestation, thus remain undetected and untreated. Malaria-infected erythrocytes may accumulate in the placenta causing adverse birth outcomes even in undetected peripheral parasitemia [6,7]. Asymptomatic malaria leads to infected erythrocytes sequestering within the placenta. The sequestration and adherence of $P$. falciparum-infected erythrocytes to the placenta is mediated by variant surface antigen 2-CSA (var2CSA), a member of P. falciparum erythrocyte membrane protein 1 (PfEMP1) which binds specifically to chondroitin sulfate A in placental cells [8]. Placental malaria infection is associated with preterm delivery, intrauterine growth restriction, stillbirths, low birth weight (LBW), and neonatal deaths $[6,9]$.

Asymptomatic malaria in pregnancy remains an important contributor of maternal anemia, the leading cause of malaria related morbidity and mortality. Additionally, asymptomatic malaria may serve as parasite reservoirs contributing to the cycle of malaria transmission $[10,11]$. The World Health Organization (WHO) recognizes asymptomatic malaria as one of the challenges for malaria elimination, and suggest that control strategies should take into account this infectious parasite reservoir [12]. The WHO recommends a protection package against malaria for all pregnant women living in endemic areas. The package includes sleeping under insecticide-treated nets, indoor spraying, effective case malaria management and intermittent preventive treatment in pregnancy with sulphadoxinepyrimethamine (IPTp-SP) [13]. Tanzania, like many other sub-Sahara African countries, has adopted and integrated the malaria control strategies recommended by WHO. IPTp involves provision of monthly single dose of sulfadoxine-pyrimethamine (SP) during ANC visit starting early in the second trimester. At least three doses ( $\geq 3 \mathrm{SP}$ ) of IPTp-SP given monthly is considered optimum during pregnancy [14]. In Tanzania, $26 \%$ of eligible pregnant women received optimal $(\geq 3)$ IPTp-SP and 56\% receive at least 2 doses of IPTp-SP in 2017 [15]. Late initiation of ANC among pregnant women is one of the reasons for low coverage of optimal IPTp-SP in Tanzania [16]. On the other hand, the reported high levels of falciparum dihydrofolate reductase (Pfdhfr) and dihydropteroate synthetase (Pfdhps) mutations and associated haplotype conferring SP resistance in Tanzania may undermine the use of IPTp-SP in improving pregnancy outcomes [17].

The Tanzanian malaria control program strives to increase coverage of insecticide-treated bed nets (ITNs), and promote effective case management as strategies to control malaria among pregnant women before they begin ANC where they receive IPTp-SP [18]. Since malaria in pregnancy is usually asymptomatic, ITNs are the main strategy for malaria control in pregnant women before and after initiation of IPTp-SP. ITNs protect against malaria both by acting as a physical barrier between mosquitoes and humans, and by repelling or killing susceptible mosquitoes. In Tanzania, mass distribution campaign and the national voucher scheme [19] have largely contributed to improved ITNs coverage to $78 \%$ in 2018 [20]. According to the recent Tanzania malaria indicator survey, the overall ITN coverage has increased to $78 \%$ [15]. However, the question remains to what extent these strategies protect pregnant women who are vulnerable to malaria before they begin ANC. Assessment of asymptomatic malaria at first antenatal clinic visit is important to evaluate the efficiency of these interventions in protecting women against malaria before the initiate of IPTp-SP.

The prevalence, severity, risk factors and treatment outcome of symptomatic malaria among pregnant women in Tanzania is investigated previously [9,21-23]. However, data on the prevalence of asymptomatic malaria and the burden of anemia among pregnant women who are not yet enrolled in the ANC are scarce in most sub-Sahara African countries including Tanzania. Evaluating the burden of asymptomatic malaria and anemia in pregnant women, particularly in high malaria transmission areas, is important to inform policymakers for targeted interventions, effective planning and implementation of malaria control programs. Therefore, this study investigated the timing of first attendance to the antenatal clinic, the burden of asymptomatic malaria and anemia, and associated risk factors at first antenatal care visits among pregnant women living in a moderate malaria transmission area in Tanzania. 


\section{Materials and Methods}

\subsection{Study Design and Population}

This was a healthcare facility-based cross-sectional study that targeted HIV-negative pregnant women attending their first ANC. Data collection took place from February 2017 to February 2018 and focused mainly on investigating the prevalence of asymptomatic malaria, anemia, and timing for the first ANC. Inclusion criteria were pregnant women who visited ANC for the first time, HIV negative, and no symptoms of malaria. The exclusion criteria were; pregnant women attending ANC for the second time or more, those started on IPTp-SP, those with a history of malaria and presumptively treated for the past one month and those who presented with symptoms related to malaria (fever, headache, chills, body ache, and joints ache). One month's history of malaria was chosen as an exclusion criterion because the target antigen (HRP2) for mRDT would still react positively for that duration after an acute malaria episode. Participants who met inclusion criteria and consented to participate were enrolled.

\subsection{Study Site}

The study was conducted at Kibiti Health Centre in Kibiti District. The district is located in the coast region of southeast Tanzania (Figure 1). The area lies south of the country's business city (Dar es Salaam) extending between $7.7218^{\circ} \mathrm{S}$ and $38.9375^{\circ} \mathrm{E}$ along the Dar es Salaam-Lindi and Mtwara highway. The district is part of hot, humid, coastal plain with varying tropical climatic conditions. The study area is characterized by seasonal rainy and vast water bodies which facilitate transmission of malaria. The region normally gets rain twice a year; the short rainy season occurs during September and October, and the longest rainfall happens from February to May. Malaria is a major public health problem in the area, accounting for the majority of out-patient health facility attendance. Malaria transmission is still endemic and common during and after the period of long rain. About $96 \%$ of all malaria cases are caused by Plasmodium falciparum, while $4 \%$ are due to P. malariae and P. ovale [24]. The prevalence of malaria in the general population is $13 \%$, according to the epidemiological characterization of malaria in the area [25].

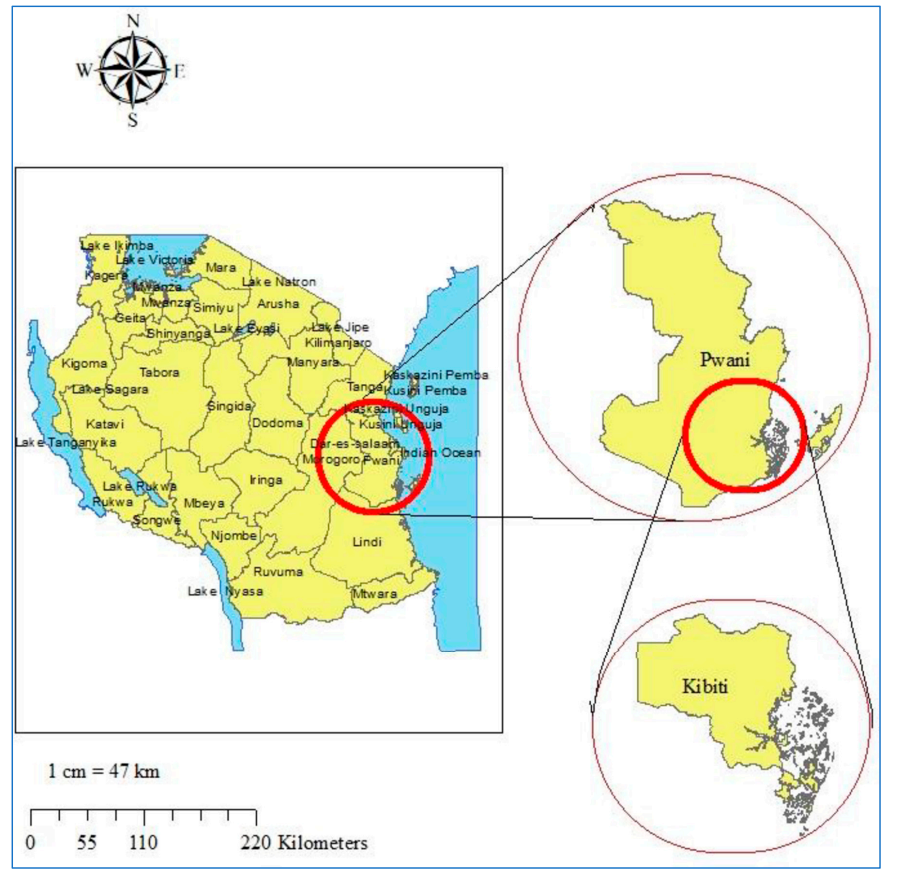

Figure 1. Study site map. Top right is the map of Coast region. Bottom right is the map of Kibiti district. On the left is the map of Tanzania. The study site map was originally generated using ArcGIS software version 10.7.1 (Esri, California, USA; https://www.esri.uconn.edu/software/arcgis-student/). 


\subsection{Data Collection Procedures}

Socio-demographics data, medical and obstetric history, the use of medication and insecticidetreated bed nets were collected using an individual case record format. Gestational age was determined by the last menstrual period (LMP) and the fundal height method, which is the standard of care in Tanzania. Weight was measured by digital weighing scale in kilograms (nearest $0.1 \mathrm{~kg}$ ). Height was measured by a potable wooden scale in centimeter (nearest $0.1 \mathrm{~cm}$ ). Temperature was recorded from the maternal armpit using a digital thermometer and fever was defined as a temperature of $\geq 37.5^{\circ} \mathrm{C}$. All recruited pregnant women were tested for HIV sero-status as a part of a routine ANC procedure. Peripheral finger-prick blood was collected for malaria detection and hemoglobin concentration determination. Asymptomatic malaria was detected by RDT (Care start, ACCESS BIO Somerset, NJ, USA) and real-time PCR (Applied Biosystems). Hemoglobin concentration was determined by HemoCue Hemoglobin 201+ analyzer (HemoCue AB Angelholm, Sweden).

\subsection{Detection of Malaria Parasites by Rapid Diagnostic Test}

Peripheral finger-prick blood was used to screen for malaria using Malaria rapid diagnostic tests (mRDTs). Malaria Pf/PAN (HRP2/PLDH) Ag combo RDTs (Care start, ACCESS BIO Somerset, NJ U.S.A) were used according to the manufacturer's instructions, and results were examined after $20 \mathrm{~min}$ following the addition of four drops of the wash buffer. The test detects four Plasmodium species responsible for malaria in human (P. falciparum, P. ovale, P. vivax and P. malariae). RDTs target P. falciparum antigens histidine-rich protein 2 (HRP2) and Plasmodium lactate dehydrogenase (pLDH) for P. falciparum, P. ovale, P. vivax and P. malariae.

\subsection{Detection of Malaria Parasites by Real-Time PCR}

Finger-prick blood samples were collected on filter papers and stored dried. Three circles of $3 \mathrm{~mm}$ each in diameter were punched from dried blood spots on filter papers to extract DNA using QIAamp DNA blood micro kit (Qiagen $\mathrm{GmbH}$, Hilden, Germany) according to the manufacturer's instructions. Screening for Plasmodium parasite species (P. falciparum, P. vivax, P. ovale, and P. malariae) was performed using a species-specific multiplex real-time PCR assay targeting the ssRNA gene as described previously $[21,26]$. The master mix for a single reaction included species-specific probes and forward primers for all four Plasmodium species used in combination with a conserved reverse primer. The P. ovale-, P. malariae-, P. vivax- and P. falciparum-probes (Applied Biosystems) were each labelled with a distinct fluorophore and Mustang Purple was used as the reference dye. The reaction was performed in duplicate in a final volume of $15 \mu \mathrm{L}$ per well containing $3 \mu \mathrm{L}$ DNA, $7.5 \mu \mathrm{L}$ of TaqMan multiplex master mix (Applied Biosystems), $0.3 \mu \mathrm{L}(10 \mu \mathrm{mol} / \mathrm{L})$ of each species-specific forward primers, $0.75 \mu \mathrm{L}$ $(10 \mu \mathrm{mol} / \mathrm{L})$ of the reverse primer, $0.15 \mu \mathrm{L}(10 \mu \mathrm{mol} / \mathrm{L})$ of each species-specific probe, passive reference dye Mustang Purple and DNA/RNA-free water. All reactions were run on 7500 Fast Real-Time PCR system (Applied Biosystems) with the following settings; each sample was initially denatured at $95^{\circ} \mathrm{C}$ for $20 \mathrm{~s}$ and cycled 45 times, with each cycle consisting of $95^{\circ} \mathrm{C}$ for $3 \mathrm{~s}$ and $60^{\circ} \mathrm{C}$ for $30 \mathrm{~s}$. Each reaction plate included four positive controls for P. falciparum, P. ovale, P. vivax, and P. malariae and a negative control with molecular-grade water instead of DNA.

\subsection{Determination of Hemoglobin Concentration}

Hemoglobin level was determined from peripheral finger prick blood by using digital HemoCue Hemoglobin 201+ analyzer (HemoCue AB Angelholm, Sweden) and expressed as g/dL. Anemia was defined when maternal hemoglobin level was $<11 \mathrm{~g} / \mathrm{dL}$. Mild, moderate and severe anemia were defined when maternal hemoglobin levels were 10-10.9 g/dL, 7-9.9 g/dL and $<7 \mathrm{~g} / \mathrm{dL}$, respectively according to the WHO guideline on the use and interpretation of haemoglobin concentrations for diagnosis of anaemia and assessment of severity in pregnant women [27]. 


\subsection{Study Outcomes}

The primary study outcomes were the prevalence of asymptomatic malaria and anemia (Hemoglobin level $<11 \mathrm{~g} / \mathrm{dL}$ ) among pregnant women at first ANC visit. Secondary outcomes were the severity of anemia according to WHO classification (mild anemia hemoglobin level, 10-10.9 g/dL; moderate anemia, hemoglobin level 7-9.9 g/dL and severe anemia hemoglobin level $<7 \mathrm{~g} / \mathrm{dL}$ ) [27]. Factors associated with malaria and anemia were determined.

\subsection{Data Management and Analysis}

Data were entered into Microsoft Excel and transferred to Statistical Package for Social Sciences (SPSS) version 20 software (IBM Corp, New York, USA) for analysis. Descriptive analysis used $n(\%)$ for binary and categorical variables and mean (SD) with $95 \% \mathrm{CI}$ or median (minimum and maximum) for continuous variables. Normality was assumed and checked for all continuous variables. The Shapiro-Wilk test $p$ values for all independent variables were $>0.05$. Visual inspection of histograms, normal Q-Q plots, and box plots showed that hemoglobin was approximately normally distributed for all independent variables with skewness and kurtosis values with their respective standard errors were all close to zero (between -1 and +1 ). Linear regressions were used to analyze independent predictors of hemoglobin concentration. Asymptomatic malaria was defined as having a positive mRDT and/or detection of malaria parasites using real-time PCR in the absence of malaria symptoms. Anemia was defined as having hemoglobin concentration below $11 \mathrm{~g} / \mathrm{dL}$. Binary logistic regressions were used to determine the predictors of asymptomatic malaria and anemia among pregnant women. Variables with a $p$-value $\leq 0.2$ in the univariate analysis were included in the multivariate model. The goodness of fit of the model was checked by Hosmer and Lemeshow test $(p>0.05)$. Crude and adjusted odds ratios with their respective $p$ values were presented. Significance level was set at 0.05 and the confidence level at $95 \%$. All $p$ values were two-sided, and a $p$ value of less than or equal to 0.05 was considered to indicate statistical significance.

\subsection{Ethics Approval}

The study was ethically approved by the Institutional Review Board of the National Institute for Medical Research (NIMR), Tanzania (NIMR/HQ/R.8a/Vol.IX/2342), and Muhimbili University of Health and Allied Sciences (MUHAS) Tanzania (2016-06-07/AEC/Vol.XI/2). Written informed consent was obtained from all study participants before commencement of the study.

\section{Results}

From February 2017 to February 2018, a total of 930 pregnant women attending their first ANC were screened for eligibility and 819 pregnant women were enrolled (Figure 2). The average gestational age at first ANC visit was 5.4 months. Eighty-five percent of enrolled participants started their first ANC visit in their second and third trimesters. Enrolled participants were mainly young adult women aged 20-34 years. About half (51.2\%) of enrolled women were pregnant for the third time and above (multigravida). The majority of enrolled women $(80.1 \%)$ reported to be using insecticide treated bed nets. Socio-demographic and obstetric characteristics of the study participants are summarized in Table 1. 


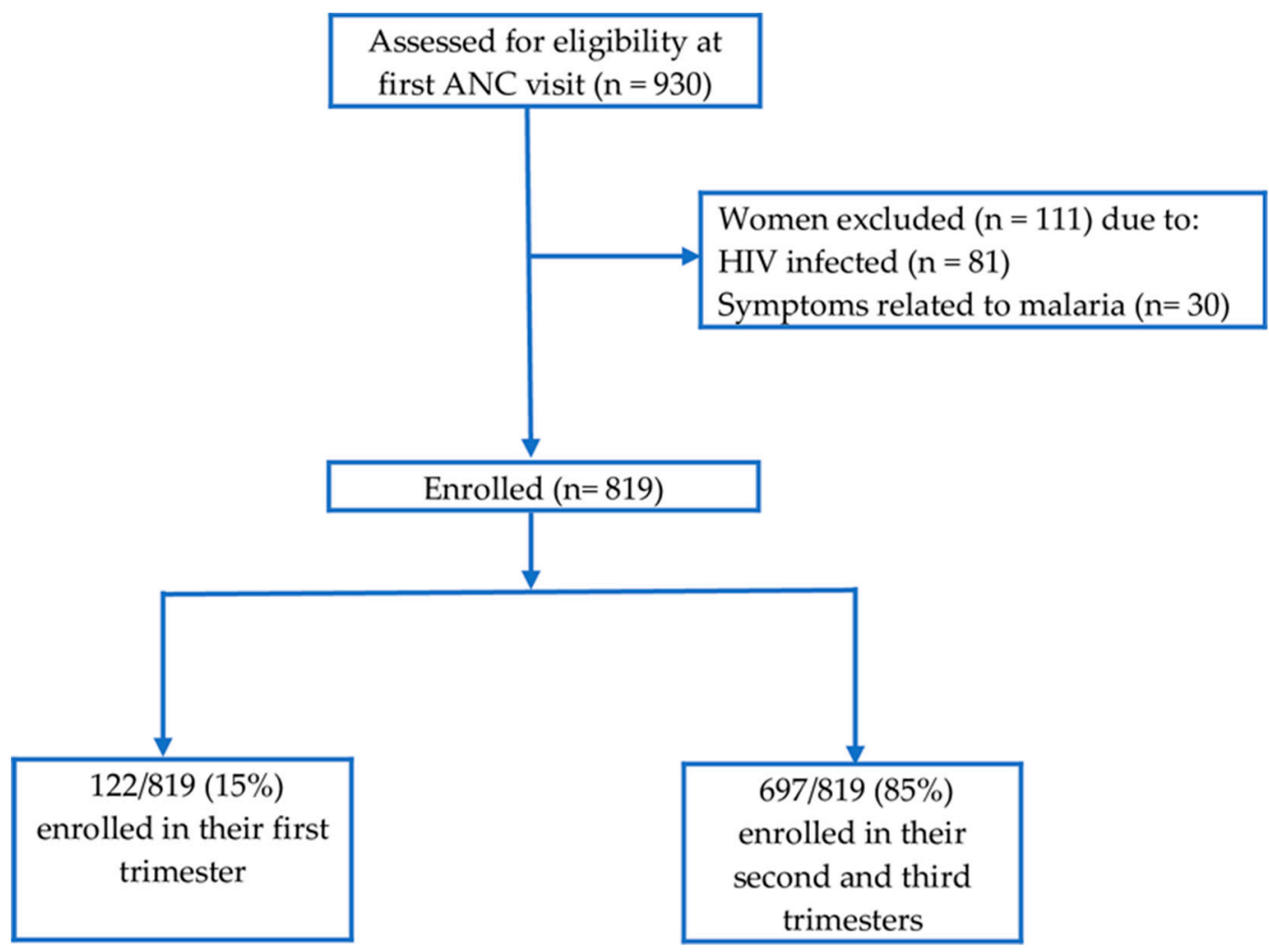

Figure 2. Participants' recruitment flow chart.

Table 1. Sociodemographic and obstetric characteristics of the study participants.

\begin{tabular}{cccc}
\hline \multicolumn{2}{c}{ Characteristics $(\boldsymbol{n}=\mathbf{8 1 9})$} & $\boldsymbol{n}$ & Frequency (\%) \\
\hline \multirow{2}{*}{ Maternal age } & Mean age (SD), years & $26.5(7.2)$ & \\
& Adolescent $(<20)$ years & 158 & 19.3 \\
& Young adult $(20-34)$ years & 519 & 63.4 \\
& Adult $(\geq 35)$ years & 142 & 17.3 \\
Gravidity & Primigravida & 219 & 26.7 \\
& Secundigravida & 181 & 22.1 \\
& Multigravida & 419 & 51.2 \\
\hline Parity (Number of live children) & Median (range) & $2(0-9)$ & 43.5 \\
\hline \multirow{2}{*}{ Gestational age } & Early ANC $(\leq 20)$ weeks & 356 & 56.5 \\
\hline \multirow{2}{*}{ Insecticide treated bed net use } & Late ANC $(\geq 21)$ weeks & 463 & 80.1 \\
& YES & 656 & 19.9 \\
\hline Height & NO & 163 & \\
\hline Weight & Median (range), cm & $151(140-168)$ & \\
\hline Body temperature & Median (range), ${ }^{\circ} \mathrm{C}$ & $37(34-37.4)$ & \\
\hline Body Mass Index (BMI) & Median (range), $\mathrm{kg} / \mathrm{cm}{ }^{2}$ & $23.8(16.4-40.7)$ & \\
\hline
\end{tabular}

\subsection{Prevalence of Asymptomatic Malaria at First Antenatal Care Visit}

Asymptomatic malaria was detected using mRDT and real-time PCR. The overall prevalence of asymptomatic malaria at first ANC visit detected by real-time PCR was 36.4\% [298/819] (95\% $\mathrm{CI}=33.1$ to 39.8$)$. On the other hand, the prevalence of asymptomatic malaria detected by $\mathrm{mRDT}$ alone was 15.4\% [126/819] (95\% CI: 12.9 to 17.9) (Figure 3A). The sensitivity and specificity of mRDT compared to real-time PCR were $43.8 \%$ and $98.7 \%$, respectively. P. falciparum mono-infection was the 
most predominant detected in $80.4 \%$ (202/251) of women who were positive by real-time PCR. Mixed infection of P. falciparum with P. malariae and P. ovale were detected in $11.2 \%(28 / 251)$ and $2 \%(5 / 251)$, respectively. Mono-infection with $P$. malariae and $P$. ovale were detected in 5.2\% (13/251) and $1.2 \%$ (3/251) women respectively (Figure 3B). P. vivax infection was not detected.

The monthly prevalence of asymptomatic malaria determined by mRDT and real-time PCR at first ANC visit remained above 25\% throughout the year (Figure 4) from February 2017 to February 2018, with higher prevalence recorded during the rainy season (February to May)

A

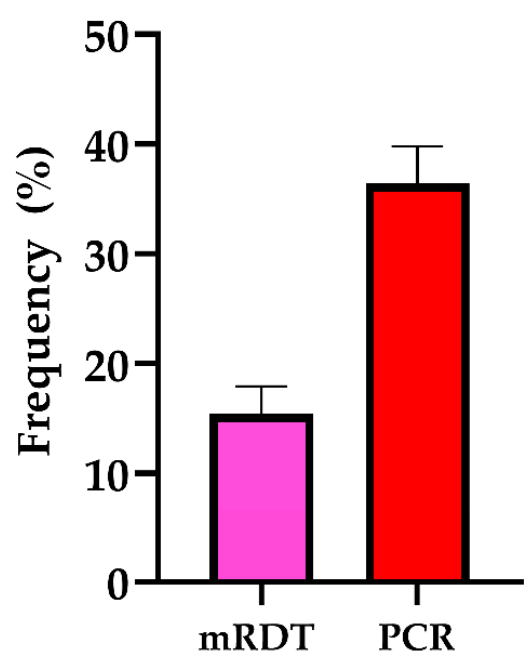

Malaria Detection Methods

B

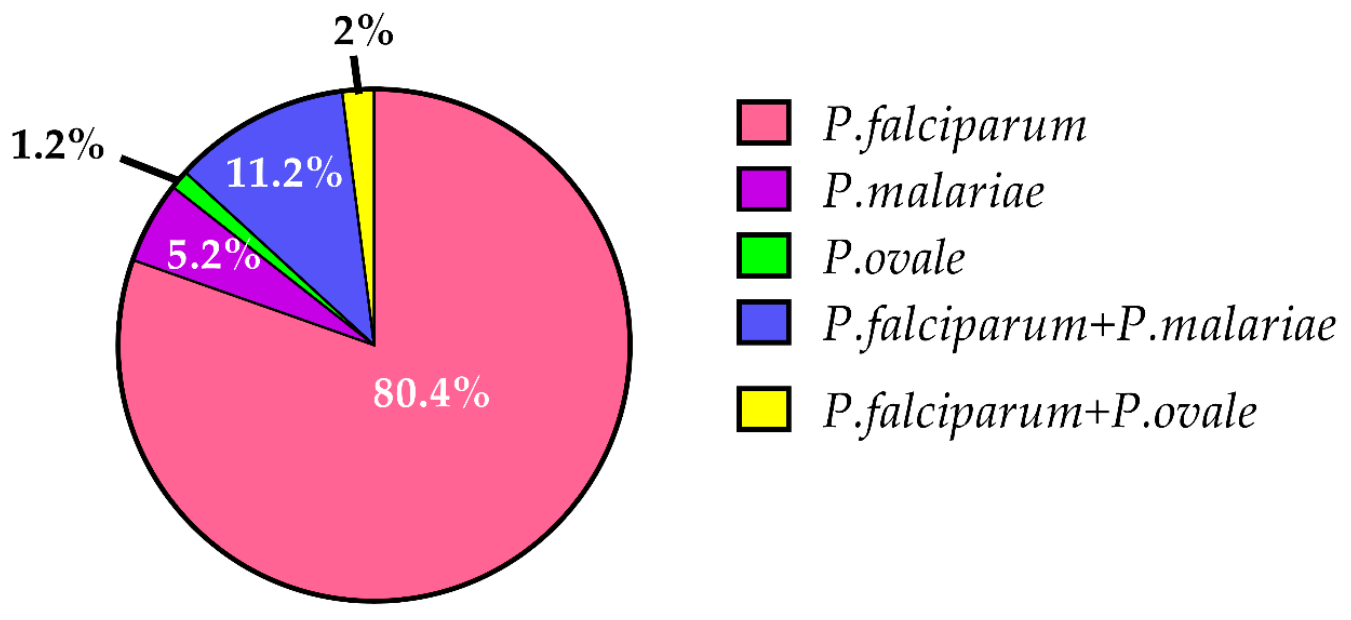

Plasimodium Species detected by PCR

Figure 3. Asymptomatic malaria among study participants: (A) overall prevalence of asymptomatic malaria detected by mRDT, real-time PCR and combined mRDT and PCR. The error bars represent 95\% confidence intervals; (B) the distribution of Plasmodium malaria parasite species detected by real-time PCR. 


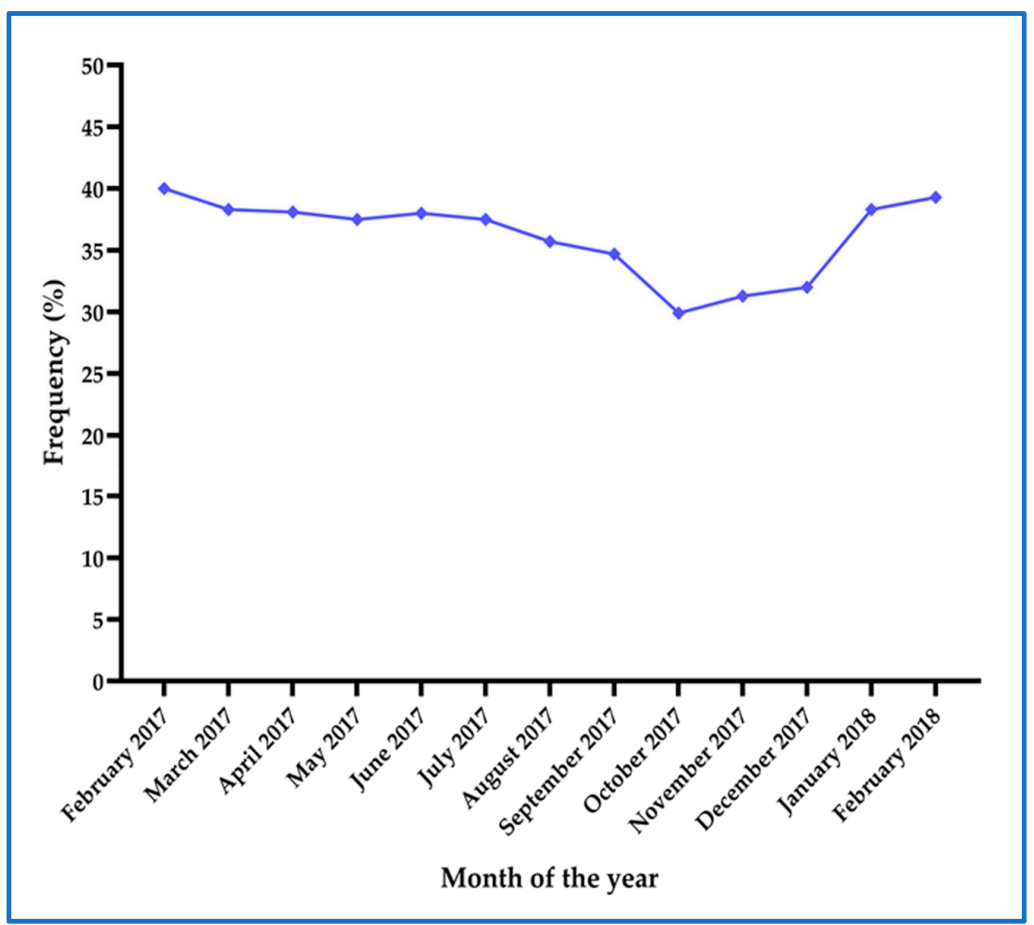

Figure 4. Prevalence of asymptomatic malaria as determined by combined mRDT and real-time PCR at each month throughout the study period.

\subsection{Factors Associated with Asymptomatic Malaria}

The association of asymptomatic malaria with various potential correlates was evaluated. Binary logistic regression analysis indicated that asymptomatic malaria was significantly associated with gravidity, maternal age, and anemia. Primigravida was associated with significantly higher odds of asymptomatic malaria compared to multigravida (Table 2). In addition, adolescence (age $<20$ years) was significantly associated with asymptomatic malaria compared to adults (age $\geq 20$ years). Moreover, asymptomatic malaria was significantly associated with moderate to severe anemia (Table 2).

Table 2. Association between asymptomatic malaria and maternal characteristics.

\begin{tabular}{|c|c|c|c|c|c|}
\hline \multirow{2}{*}{ Characteristic } & \multirow{2}{*}{$\begin{array}{c}\text { Asymptomatic Malaria } \\
n / N(\%)\end{array}$} & \multicolumn{2}{|c|}{ Univariate Analysis } & \multicolumn{2}{|c|}{ Multivariate Analysis } \\
\hline & & OR $(95 \% \mathrm{CI})$ & $p$ Value & aOR $(95 \% \mathrm{CI})$ & $p$ Value \\
\hline \multicolumn{6}{|l|}{ Age } \\
\hline Adolescent (16-19 years) & $73 / 158(46.2)$ & $1.66(1.17,2.37)$ & 0.005 & $1.48(0.94,2.35)$ & 0.09 \\
\hline Adult ( $\geq 20$ years) & $225 / 661(34)$ & 1 & & 1 & \\
\hline \multicolumn{6}{|l|}{ Gravidity } \\
\hline Primigravida & $94 / 219(42.9)$ & $1.46(1.06,2.00)$ & & $0.92(0.85,1.00)$ & 0.06 \\
\hline Multigravida & $204 / 600(34)$ & 1 & 0.019 & 1 & \\
\hline \multicolumn{6}{|l|}{ Gestational age (Weeks) } \\
\hline $\begin{array}{c}\text { Early attendance to ANC } \\
\text { ( } \leq 20 \text { weeks) }\end{array}$ & $135 / 356(37.9)$ & $1.12(0.84,1.50)$ & 0.42 & & \\
\hline $\begin{array}{l}\text { Late attendance to ANC } \\
\text { ( } \geq 21 \text { weeks })\end{array}$ & $163 / 463(35.2)$ & 1 & & & \\
\hline \multicolumn{6}{|l|}{ ITN use } \\
\hline YES & $234 / 656(35.7)$ & $0.85(0.60,1.21)$ & & & \\
\hline $\mathrm{NO}$ & $64 / 163(39.2)$ & 1 & 0.36 & & \\
\hline${ }^{*}$ BMI median (range) $\mathrm{kg} / \mathrm{m}^{2}$ & $23.8(16.4-40.7)$ & $0.98(0.94,1.02)$ & 0.31 & & \\
\hline \multicolumn{6}{|l|}{ Anemia } \\
\hline Severe \& moderate anemia & $129 / 315(41)$ & $1.49(1.05,2.10)$ & 0.024 & $1.39(0.98,1.97)$ & 0.07 \\
\hline Mild anemia & $87 / 246(35.4)$ & $1.17(0.81,1.70)$ & 0.40 & $1.13(0.78,1.64)$ & 0.51 \\
\hline No anemia & $82 / 258(31.8)$ & 1 & & 1 & \\
\hline
\end{tabular}

$\mathrm{BMI}=$ Body mass index; ITN = insecticide treated bed net; $\mathrm{CI}=$ Confidence Interval; ${ }^{*}$ Continuous variable; $\mathrm{OR}=$ crude odds ratio; aOR = adjusted odds ratio. 


\subsection{Prevalence of Anemia at First Antenatal Care Visit}

The overall prevalence of anemia among pregnant women at first ANC visit was $68.5 \%$ [561/819] $(95 \% \mathrm{CI}=65.2$ to 71.6$)$. Majority of participants $(54.4 \%$ [305/561]) had moderate anemia (hemoglobin = 7-9.9 g/dL) followed by mild anemia 43.9\% [246/561] (hemoglobin $=10-10.9 \mathrm{~g} / \mathrm{dL})$ and only a few pregnant women $(1.7 \%$ [10/561]) had severe anemia (hemoglobin < $<$ g/dL) (Figure 5).

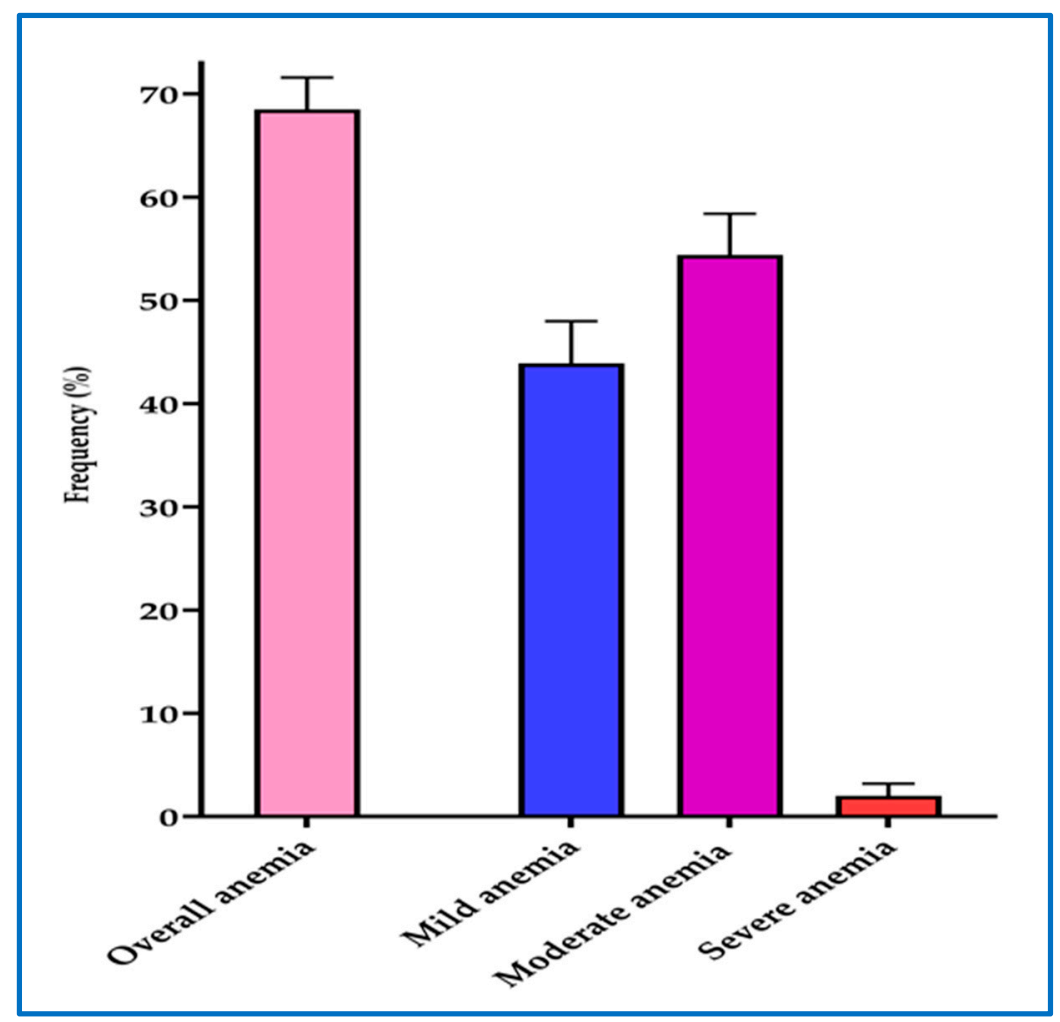

Figure 5. Overall prevalence and severity of anemia among pregnant women. The error bars represent $95 \%$ confidence intervals. Mild anemia $(\mathrm{Hb}=10-10.9 \mathrm{~g} / \mathrm{dL})$, Moderate anemia $(\mathrm{Hb}=7-9.9 \mathrm{~g} / \mathrm{dL})$, Severe anemia $(\mathrm{Hb}<7 \mathrm{~g} / \mathrm{dL})$.

\subsection{Factors Associated with Anemia}

Potential predictors of anemia at first ANC visit were investigated using binary logistic regression. Maternal age, gravidity, and late initiation of ANC (gestational age $\geq 21$ weeks) were significantly associated with anemia at first ANC visit. Adolescence (age $<20$ years) was significantly associated with higher odds of anemia compared to adults (age $\geq 20$ years) (Table 3 ). On the other hand, primigravida was significantly associated with higher odds of anemia compared to multigravida. In the multivariate binary logistic model, primigravida and late attendance to ANC (gestational age $\geq 21$ weeks) were significantly associated with higher odds of anemia (Table 3). 
Table 3. Factors associated with anemia among pregnant women.

\begin{tabular}{|c|c|c|c|c|c|}
\hline \multirow{2}{*}{ Characteristic } & \multirow{2}{*}{$\begin{array}{l}\text { Anemia } \\
n / N(\%)\end{array}$} & \multicolumn{2}{|c|}{ Univariate Analysis } & \multicolumn{2}{|c|}{ Multivariate Analysis } \\
\hline & & OR $(95 \% \mathrm{CI})$ & $p$ Value & aOR $(95 \% \mathrm{CI})$ & $p$ Value \\
\hline \multicolumn{6}{|l|}{ Age } \\
\hline Adolescent (16-19 years) & $127 / 158(80.4)$ & $2.14(1.40,3.28)$ & & $1.35(0.78,2.32)$ & \\
\hline Adult $(\geq 20$ years $)$ & $434 / 661(65.7)$ & 1 & $<0.001$ & 1 & 0.28 \\
\hline \multicolumn{6}{|l|}{ Gravidity } \\
\hline Primigravida & 176/219 (80.4) & $2.29(1.57,3.32)$ & & $1.96(1.22,3.13)$ & \\
\hline Multigravida & $385 / 600(64.4)$ & 1 & $<0.001$ & 1 & 0.005 \\
\hline \multicolumn{6}{|l|}{ Gestational age } \\
\hline Early attendance to ANC ( $\leq 20$ weeks) & $35 / 356(66)$ & $0.82(0.61,1.10)$ & & $0.72(0.53,0.98)$ & \\
\hline Late attendance to ANC ( $\geq 21$ weeks) & $326 / 463(70.4)$ & 1 & 0.18 & 1 & 0.035 \\
\hline \multicolumn{6}{|l|}{ ITN use } \\
\hline YES & $448 / 656(68.3)$ & $0.96(0.67,1.39)$ & & & \\
\hline NO & $113 / 163(69.3)$ & 1 & 0.84 & & \\
\hline${ }^{*}$ BMI median (range) kg/m² & $23.8(16.4-40.7)$ & $0.97(0.93,1.01)$ & 0.11 & $0.97(0.93,1.01)$ & 0.19 \\
\hline
\end{tabular}

\subsection{Factors Associated with Hemoglobin Concentration}

The overall mean hemoglobin concentration with one standard deviation was 10.3 (1.4) g/dL. Various factors associated with hemoglobin concentration were evaluated using linear regression model. Hemoglobin concentration was significantly associated with asymptomatic malaria, gravidity, body mass index (BIM) and maternal age (Table 4). Asymptomatic malaria was significantly associated with reduced hemoglobin concentration. In addition, multigravida was associated with significantly higher hemoglobin concentration compared to primigravida. Moreover, an increase in one unit $\left(\mathrm{kg} / \mathrm{m}^{2}\right) \mathrm{BMI}$ and an increase in maternal age by one year were significantly associated with increased hemoglobin concentrations (Table 4). In the multivariate regression model, primigravida and low BMI were significantly associated with low hemoglobin concentration (Table 4).

Table 4. Linear regression analysis for determinants of hemoglobin concentration (g/dL) among pregnant women.

\begin{tabular}{|c|c|c|c|c|c|c|}
\hline \multirow[b]{2}{*}{ Characteristic } & \multicolumn{3}{|c|}{ Univariate Analysis } & \multicolumn{3}{|c|}{ Multivariate Analysis } \\
\hline & $\begin{array}{l}\text { Beta Coefficient } \\
{[95 \% \mathrm{CI}]}\end{array}$ & $t$ & $p$ Value & $\begin{array}{c}\text { Beta Coefficient } \\
{[95 \% \mathrm{CI}]}\end{array}$ & $t$ & $p$ Value \\
\hline Age (Years) & $0.03(0.02,0.04)$ & 4.36 & $<0.001$ & $0.01(-0.01,0.02)$ & 0.88 & 0.38 \\
\hline * Gravidity & & & $<0.001$ & & & \\
\hline Multigravida & $0.64(0.42,0.86)$ & 5.77 & & $0.53(0.27,0.80)$ & 3.95 & $<0.001$ \\
\hline Primigravida & 1 & & & 1 & & \\
\hline Gestational age (Weeks) & $-0.01(-0.03,0.02)$ & -0.27 & 0.79 & & & \\
\hline${ }^{*}$ ITN use & & & 0.96 & & & \\
\hline YES & 1 & & & & & \\
\hline NO & $-0.01(-0.25,0.24)$ & -0.05 & & & & \\
\hline BMI & $0.04(0.01,0.07)$ & 3.05 & 0.002 & $0.03(0.01,0.06)$ & 2.49 & 0.013 \\
\hline * Asymptomatic Malaria & & & 0.025 & & & \\
\hline YES & $-0.23(-0.44,-0.03)$ & & & $-0.17(-0.37,0.03)$ & & \\
\hline $\mathrm{NO}$ & 1 & -2.25 & & 1 & 1.65 & 0.09 \\
\hline
\end{tabular}

$\mathrm{BMI}=$ Body mass index; ITN = insecticide treated bed net; CI = Confidence Interval; * Categorical variable; $t=$ student $t$-test.

\section{Discussion}

This study assessed the prevalence and risk factors of asymptomatic malaria and anemia at first ANC visit among HIV negative pregnant women living in a moderate malaria transmission area of Tanzania. The major findings include: (i) a high prevalence of asymptomatic malaria (36.4\%) at the 
first antenatal care visit, which sustained to be $>25 \%$ throughout the year during the study period, (ii) an overall high prevalence of anemia $(>60 \%$ ), (iii) delayed presentation for the first antenatal care (5.4 months) and (iv) primigravida and younger maternal age as risk factors for asymptomatic malaria, and anemia at first ANC visit. The prevalence of asymptomatic malaria (36.4\%) at first ANC before initiating IPTp-SP found among pregnant women in the study area is higher than the prevalence of asymptomatic malaria at first ANC reported from Kenya (35\%) [28], but lower than that reported in Uganda (50\%) [29] and Malawi (54.2\%) [30]. The difference in the burden of asymptomatic malaria at first ANC visit could be due to different malaria transmission intensities between different countries. Nevertheless, this finding provides further evidence that asymptomatic malaria is still a public health problem among pregnant women in endemic countries.

The high prevalence of asymptomatic malaria among pregnant women found in this study could indicate the challenge with the current malaria prevention strategies during early pregnancy before initiating the IPTp-SP. In Tanzania, the national malaria control program strives to expand ITNs coverage which is the key strategy to prevent malaria. However, utilization of ITNs among pregnant women in Tanzania has declined from 75\% in 2011 to 51\% in 2017 [15,31]. While the impact of INTs in preventing malaria in sub-Saharan Africa is well known [32], the declining trend in its usage among pregnant women and outdoor mosquito biting could be the reasons for the detected high prevalence of asymptomatic malaria among pregnant women in the study area. On the other hand, factors such as poor sanitation and agricultural activities commonly practiced along water sources may favor breeding of vectors, thus facilitating malaria transmission in the setting. The use of mass media to improve awareness, especially on behavior change related to sanitation and ITNs use may help to reduce the burden. Other strategies such as indoor residual spray have been reported to be effective for the prevention of malaria in sub-Saharan Africa [33].

The prevalence of asymptomatic malaria among pregnant women during their first ANC visit was consistently above $25 \%$ throughout the study year. The highest prevalence (40\%) was recorded in February, indicating high transmission during the rainy season. The persistent prevalence of asymptomatic malaria suggests the stable transmission in the study area. The overall prevalence of asymptomatic malaria in pregnant women found in this study is five-fold higher than the current overall prevalence of symptomatic malaria in Tanzania 7.3\% [20]. Currently, the national ANC protocol recommends the use of IPTp-SP for all asymptomatic pregnant women from the early second trimester and effective symptomatic case treatment. However, the high burden of asymptomatic malaria found in this study indicate the need to integrate screening and treatment of asymptomatic malaria at first ANC which is currently not commonly practiced in the study area. The prevalence of asymptomatic malaria determined by the current diagnostic standard of care in Tanzania (RDTs) (15.4\%) was also two-fold higher than the national prevalence of symptomatic malaria [20]. This finding suggests that despite the limited sensitivity of mRDT compared to PCR, the use of RDTs for screening and treatment at first ANC could help to reduce the burden of asymptomatic malaria among pregnant women.

In sub-Saharan Africa, malaria continues to be the main reason for low birth weight which is the risk factor for poor neurodevelopment and infant mortality [34]. For instance, in 2018, 16\% of all children born with LBW were associated to malaria exposure during pregnancy [35]. Exposure to asymptomatic malaria during the second and third trimesters may result in placental infection and leading to LBW [36]. Thus, the high burden of asymptomatic malaria found in this study suggests the need to strengthen control of malaria in pregnancy in order to improve maternal and newborn health. The study results indicate that primigravida and adolescent pregnant women were more vulnerable to asymptomatic malaria. The reason for primigravida having a higher risk and burden of asymptomatic malaria than multigravida could be related to parity level immunity to variant surface antigen of P. falciparum erythrocyte membrane protein 1 (PfEMP1), which is acquired through consecutive pregnancies [37]. On the other hand, the significantly higher odds of asymptomatic malaria among adolescent pregnant women compared to adult women may be related to immunity associated with the increasing maternal age. 
High overall prevalence of anemia (68.5\%) among pregnant women was found in this study. Majority of participants had moderate to mild anemia. Only few pregnant women were found to have severe anemia at first ANC visit. Pregnant women with asymptomatic malaria had significantly higher odds of moderate to severe anemia compared to those without detected malaria parasites. In addition, asymptomatic malaria was significantly associated with low hemoglobin concentration. A study in Cameroon, reported that both asymptomatic and symptomatic malaria at first ANC visit were associated with low hemoglobin levels and anemia [38]. Therefore, our finding adds evidence to further support the association of asymptomatic malaria with anemia during pregnancy. Malaria in pregnancy may causes anemia through hemolysis, increased splenic clearance of erythrocytes, and reduced red blood cell production [39]. Malaria associated maternal anemia is the leading cause of morbidity and mortality associated with malaria in pregnancy [35].

Factors such as poor nutrition could be among the reasons for high anemia in the study area. In this study, BMI was associated with significantly low hemoglobin concentration. Although caution is needed when relating BMI with nutritional status especially during pregnancy, this finding may suggest that poor nutrition could be one of the reasons for the high anemia burden in the study area. Additionally, primigravida and adolescent pregnant women were found to have significantly higher odds of anemia compared to multigravida and adult pregnant women, respectively. The high risk of anemia among primigravida and adolescent women may be related to their higher susceptibility to asymptomatic malaria. Anemia prevalence found in this study (68.5\%) is higher than that reported in Nigeria (59.6\%), Southeast Ethiopia (27.9\%) and in low malaria area of northern Tanzania (23\%) [40-42], indicating that anemia is a major public health burden among pregnant women in the study area.

Maternal anemia during pregnancy could lead to adverse birth outcomes such as preterm birth and LBW [43-45]. In addition, anemia during pregnancy may cause fetal iron deficiency which is an established risk factor for poor neurodevelopment [46]. Iron is an essential requirement in fetal synaptogenesis and myelination, thus its deficiency could be the reason for poor neurodevelopment $[47,48]$. Poor neurodevelopment might produce long-lasting defects in mental development and performance that may further impair child learning capacity. Strategies such as early initiation of ANC and effective control of malaria during pregnancy should be strengthened.

The median gestational age at first ANC visit was 5.4 months. The majority of pregnant women in the study area $(85 \%)$ appeared to their first ANC visit in the late second and early third trimesters, which is similar to findings from other developing countries [3,4]. Usually, visits to the ANC serve as a routine standard of care platform for delivery of IPTp-SP and iron/folic acid supplementation for prevention of malaria and anemia, respectively. Late presentation to the ANC indicates that pregnant women may not fully benefit from preventive and curative services that are provided in the ANC. Our results indicate that women who initiated ANC late (gestational age $\geq 21$ weeks) had significantly higher burden of anemia compared to those who started ANC early (gestational age $<20$ weeks). This finding suggest that early initiation of ANC could help to reduce the burden of anemia. Furthermore, anemia diagnosed at the late second trimester might not be corrected by iron/folic acid supplementation to term [45]. Hence sensitization of pregnant women to begin ANC visits early in the first trimester could help to improve public health among pregnant women.

Our findings may also serve as an evaluation for the efficiency of existing interventions for the prevention of malaria and anemia before ANC initiation. The results from this study indicate the need to strengthen and expand the ITNs coverage and usage and screening for malaria and anemia at first ANC visit, particularly for those pregnant women living in a high malaria transmission area.

The study had some limitations. As it was conducted in an area of moderate malaria transmission intensity $(<50 \%)$, the results of this study may not be generalized to areas of lower $(<5 \%)$ and higher $(>50 \%)$ malaria transmission intensities. Additionally, the study assessed only asymptomatic malaria in pregnancy. Excluding pregnant women with symptomatic malaria might be underestimating the overall burden of malaria at first ANC visit in the study area. However, the strength of this study is that the data collection spans over one year, covering both high and low malaria seasons. Moreover, the use 
of sensitive techniques (real-time PCR) helped to detect malaria parasitemia missed by mRDT which has limited sensitivity; thus, the findings of this study may reflect the true burden of asymptomatic malaria in the study area.

\section{Conclusions}

We report delayed presentation in seeking ANC, and a high prevalence of asymptomatic malaria and anemia among pregnant women at first ANC visit. Primigravida and younger maternal age significantly correlate with both asymptomatic malaria and anemia. Asymptomatic malaria and low BMI are significant predictors of low hemoglobin concentration. Screening for malaria and anemia, particularly in primigravida and younger mothers, during the first ANC visit is recommended for targeted interventions. Strengthening ITNs coverage and screening for malaria and anemia during first antenatal care, particularly in areas with high malaria transmission is recommended for targeted intervention and care. Sensitization for earlier ANC initiation and educating women on nutrition and diets during ANC visit is recommended to reduce the burden of anemia. In addition, new strategies to outreach pregnant women who have not yet started ANC are recommended to maximize the benefit from the service and alleviate the burden of malaria and anemia in pregnancy.

Author Contributions: Conceptualization, E.M.M., E.A., A.A.R.K., and O.M.; methodology, E.M.M., E.A., A.A.R.K., and O.M.; formal analysis, E.M.M., E.A., A.A.R.K., and O.M.; writing-original draft preparation, E.M.; writing-review and editing, E.M.M., E.A., A.A.R.K., and O.M. All authors have read and agreed to the published version of the manuscript.

Funding: This research was funded by the Swedish International Development Cooperation Agency-Sida to Muhimbili University of Health and Allied Sciences (Grant number-51170072).

Acknowledgments: We thank the Swedish International Development Cooperation Agency (Sida) through Malaria and Neglected Tropical Diseases Sub-programme at Muhimbili University of Health and Allied Sciences for providing financial support for this study. We also convey our sincere thanks to the study participants, the District medical officer Kibiti district, the medical officer in charge of Kibiti health center, nurses and clinicians at the antenatal care clinic and laboratory technicians at Kibiti health care center for their support and participation in the study.

Conflicts of Interest: The authors declare no conflict of interest.

\section{References}

1. McLean, E.; Cogswell, M.; Egli, I.; Wojdyla, D.; de Benoist, B. Worldwide prevalence of anaemia, WHO Vitamin and Mineral Nutrition Information System, 1993-2005. Public Health Nutr. 2009, 12, $444-454$. [CrossRef]

2. Amek, N.O.; Van Eijk, A.; Lindblade, K.A.; Hamel, M.; Bayoh, N.; Gimnig, J.; Laserson, K.F.; Slutsker, L.; Smith, T.; Vounatsou, P. Infant and child mortality in relation to malaria transmission in KEMRI/CDC HDSS, Western Kenya: Validation of verbal autopsy. Malar. J. 2018, 17, 37. [CrossRef] [PubMed]

3. Ebeigbe, P.N.; Igberase, G.O. Reasons given by pregnant women for late initiation of antenatal care in the niger delta, Nigeria. Ghana Med. J. 2010, 44, 47-51. [PubMed]

4. Gebremeskel, F.; Dibaba, Y.; Admassu, B. Timing of first antenatal care attendance and associated factors among pregnant women in Arba Minch Town and Arba Minch District, Gamo Gofa Zone, south Ethiopia. J. Environ. Public Health 2015, 2015, 971506. [CrossRef]

5. van Eijk, A.M.; Hill, J.; Noor, A.M.; Snow, R.W.; ter Kuile, F.O. Prevalence of malaria infection in pregnant women compared with children for tracking malaria transmission in sub-Saharan Africa: A systematic review and meta-analysis. Lancet Glob. Health 2015, 3, e617-e628. [CrossRef]

6. Cohee, L.M.; Kalilani-Phiri, L.; Boudova, S.; Joshi, S.; Mukadam, R.; Seydel, K.B.; Mawindo, P.; Thesing, P.; Kamiza, S.; Makwakwa, K.; et al. Submicroscopic malaria infection during pregnancy and the impact of intermittent preventive treatment. Malar. J. 2014, 13, 274. [CrossRef]

7. Kalilani-Phiri, L.; Thesing, P.C.; Nyirenda, O.M.; Mawindo, P.; Madanitsa, M.; Membe, G.; Wylie, B.; Masonbrink, A.; Makwakwa, K.; Kamiza, S.; et al. Timing of malaria infection during pregnancy has characteristic maternal, infant and placental outcomes. PLoS ONE 2013, 8, e74643. [CrossRef] 
8. $\quad$ Salanti, A.; Dahlback, M.; Turner, L.; Nielsen, M.A.; Barfod, L.; Magistrado, P.; Jensen, A.T.; Lavstsen, T.; Ofori, M.F.; Marsh, K.; et al. Evidence for the involvement of VAR2CSA in pregnancy-associated malaria. J. Exp. Med. 2004, 200, 1197-1203. [CrossRef]

9. Mlugu, E.M.; Minzi, O.; Asghar, M.; Farnert, A.; Kamuhabwa, A.A.R.; Aklillu, E. Effectiveness of Sulfadoxine-Pyrimethamine for Intermittent Preventive Treatment of Malaria and Adverse Birth Outcomes in Pregnant Women. Pathogens 2020, 9, 207. [CrossRef]

10. Sumari, D.; Mwingira, F.; Selemani, M.; Mugasa, J.; Mugittu, K.; Gwakisa, P. Malaria prevalence in asymptomatic and symptomatic children in Kiwangwa, Bagamoyo district, Tanzania. Malar. J. 2017, 16, 222. [CrossRef]

11. Steenkeste, N.; Rogers, W.O.; Okell, L.; Jeanne, I.; Incardona, S.; Duval, L.; Chy, S.; Hewitt, S.; Chou, M.; Socheat, D.; et al. Sub-microscopic malaria cases and mixed malaria infection in a remote area of high malaria endemicity in Rattanakiri province, Cambodia: Implication for malaria elimination. Malar. J. 2010, 9, 108. [CrossRef] [PubMed]

12. World Health Organization. Global Technical Strategy for Malaria 2016-2030. 2015. Available online: https://www.who.int/malaria/publications/atoz/9789241564991/en/ (accessed on 10 March 2020).

13. World Health Organization. WHO Evidence Review Group: Intermittent Preventive Treatment of Malaria in Pregnancy (IPTp) with Sulfadoxine-Pyrimethamine (SP). Available online: https://www.who.int/malaria/ mpac/sep2012/iptp_sp_erg_meeting_report_july2012.pdf (accessed on 1 December 2019).

14. Kayentao, K.; Garner, P.; van Eijk, A.M.; Naidoo, I.; Roper, C.; Mulokozi, A.; MacArthur, J.R.; Luntamo, M.; Ashorn, P.; Doumbo, O.K.; et al. Intermittent preventive therapy for malaria during pregnancy using 2 vs 3 or more doses of sulfadoxine-pyrimethamine and risk of low birth weight in Africa: Systematic review and meta-analysis. JAMA 2013, 309, 594-604. [CrossRef] [PubMed]

15. Ministry of Health, Community Development, Gender, Elderly and Children, Ministry of Health Zanzibar, National Bureau of Statistics, Office of Chief Government Statistician and ICF. Tanzania Malaria Indicator Survey 2017. Available online: https://hsprogram.com/pubs/pdf/MIS31/MIS31.pdf (accessed on 4 December 2019).

16. Exavery, A.; Mbaruku, G.; Mbuyita, S.; Makemba, A.; Kinyonge, I.P.; Kweka, H. Factors affecting uptake of optimal doses of sulphadoxine-pyrimethamine for intermittent preventive treatment of malaria in pregnancy in six districts of Tanzania. Malar. J. 2014, 13, 22. [CrossRef] [PubMed]

17. Baraka, V.; Ishengoma, D.S.; Fransis, F.; Minja, D.T.R.; Madebe, R.A.; Ngatunga, D.; Van Geertruyden, J.-P. High-level Plasmodium falciparum sulfadoxine-pyrimethamine resistance with the concomitant occurrence of septuple haplotype in Tanzania. Malar. J. 2015, 14, 439. [CrossRef]

18. Ministry of Health and Social Welfare. National Malaria Strategic Plan 2014-2020. Dar es Salaam: Ministry of Health and Social Welfare. 2014. Available online: https://www.out.ac.tz/wp-content/uploads/2019/10/ Malaria-Strategic-Plan-2015-2020-1.pdf (accessed on 3 March 2020).

19. Kramer, K.; Mandike, R.; Nathan, R.; Mohamed, A.; Lynch, M.; Brown, N.; Mnzava, A.; Rimisho, W.; Lengeler, C. Effectiveness and equity of the Tanzania National Voucher Scheme for mosquito nets over 10 years of implementation. Malar. J. 2017, 16, 255. [CrossRef]

20. President's Malaria Initiative Tanzania-Malaria Operational Plan FY 2019. Available online: https://www.pmi.gov/docs/default-source/default-document-library/malaria-operational-plans/fy19/fy2019-tanzania-malaria-operational-plan.pdf?sfvrsn=3 (accessed on 4 February 2020).

21. Mutagonda, R.F.; Minzi, O.M.S.; Massawe, S.N.; Asghar, M.; Farnert, A.; Kamuhabwa, A.A.R.; Aklillu, E. Pregnancy and CYP3A5 Genotype Affect Day 7 Plasma Lumefantrine Concentrations. Drug Metab. Dispos. 2019, 47, 1415-1424. [CrossRef]

22. Mutagonda, R.F.; Kamuhabwa, A.A.; Minzi, O.M.; Massawe, S.N.; Maganda, B.A.; Aklillu, E. Malaria prevalence, severity and treatment outcome in relation to day 7 lumefantrine plasma concentration in pregnant women. Malar. J. 2016, 15, 278. [CrossRef]

23. Mutagonda, R.F.; Kamuhabwa, A.A.R.; Minzi, O.M.S.; Massawe, S.N.; Asghar, M.; Homann, M.V.; Farnert, A.; Aklillu, E. Effect of pharmacogenetics on plasma lumefantrine pharmacokinetics and malaria treatment outcome in pregnant women. Malar. J. 2017, 16, 267. [CrossRef] 
24. Ministry of Health, Community Development, Gender, Elderly and Children (MoHCDGEC) [TanzaniaMainland], Ministry of Health (MoH) [Zanzibar]. Tanzania Malaria Operational Plan FY 2015. Available online: https://www.pmi.gov/docs/default-source/default-document-library/malaria-operationalplans/fy-15/fy-2015-tanzania-malaria-operational-plan.pdf (accessed on 1 December 2019).

25. Khatib, R.A.; Chaki, P.P.; Wang, D.Q.; Mlacha, Y.P.; Mihayo, M.G.; Gavana, T.; Xiao, N.; Zhou, X.N.; Abdullah, S. Epidemiological characterization of malaria in rural southern Tanzania following China-Tanzania pilot joint malaria control baseline survey. Malar. J. 2018, 17, 292. [CrossRef]

26. Shokoples, S.E.; Ndao, M.; Kowalewska-Grochowska, K.; Yanow, S.K. Multiplexed real-time PCR assay for discrimination of Plasmodium species with improved sensitivity for mixed infections. J. Clin. Microbiol. 2009, 47, 975-980. [CrossRef]

27. World Health Organization. Haemoglobin Concentrations for the Diagnosis of Anaemia and Assessment of Severity. Available online: https://apps.who.int/iris/handle/10665/85839 (accessed on 1 December 2019).

28. Desai, M.; Gutman, J.; L'Lanziva, A.; Otieno, K.; Juma, E.; Kariuki, S.; Ouma, P.; Were, V.; Laserson, K.; Katana, A.; et al. Intermittent screening and treatment or intermittent preventive treatment with dihydroartemisinin-piperaquine versus intermittent preventive treatment with sulfadoxine-pyrimethamine for the control of malaria during pregnancy in western Kenya: An open-label, three-group, randomised controlled superiority trial. Lancet 2015, 386, 2507-2519.

29. Kakuru, A.; Jagannathan, P.; Muhindo, M.K.; Natureeba, P.; Awori, P.; Nakalembe, M.; Opira, B.; Olwoch, P.; Ategeka, J.; Nayebare, P.; et al. Dihydroartemisinin-Piperaquine for the Prevention of Malaria in Pregnancy. N. Engl. J. Med. 2016, 374, 928-939. [CrossRef]

30. Madanitsa, M.; Kalilani, L.; Mwapasa, V.; van Eijk, A.M.; Khairallah, C.; Ali, D.; Pace, C.; Smedley, J.; Thwai, K.L.; Levitt, B.; et al. Scheduled Intermittent Screening with Rapid Diagnostic Tests and Treatment with Dihydroartemisinin-Piperaquine versus Intermittent Preventive Therapy with Sulfadoxine-Pyrimethamine for Malaria in Pregnancy in Malawi: An Open-Label Randomized Controlled Trial. PLoS Med. 2016, 13, e1002124.

31. Ministry of Health, Community Development, Gender, Elderly and Children, Ministry of Health Zanzibar, National Bureau of Statistics, Office of Chief Government Statistician and ICF. Tanzania Demographic and Health Survey and Malaria Indicator Survey 2015-2016 Final Report. Available online: https://dhsprogram. com/publications/publication-fr321-dhs-final-reports.cfm (accessed on 4 December 2019).

32. Bhatt, S.; Weiss, D.J.; Cameron, E.; Bisanzio, D.; Mappin, B.; Dalrymple, U.; Battle, K.E.; Moyes, C.L.; Henry, A.; Eckhoff, P.A.; et al. The effect of malaria control on Plasmodium falciparum in Africa between 2000 and 2015. Nature 2015, 526, 207-211. [CrossRef]

33. Sherrard-Smith, E.; Griffin, J.T.; Winskill, P.; Corbel, V.; Pennetier, C.; Djenontin, A.; Moore, S.; Richardson, J.H.; Muller, P.; Edi, C.; et al. Systematic review of indoor residual spray efficacy and effectiveness against Plasmodium falciparum in Africa. Nat. Commun. 2018, 9, 4982. [CrossRef]

34. Chaponda, E.B.; Chandramohan, D.; Michelo, C.; Mharakurwa, S.; Chipeta, J.; Chico, R.M. High burden of malaria infection in pregnant women in a rural district of Zambia: A cross-sectional study. Malar. J. 2015, 14, 380. [CrossRef]

35. World Health organization. World Malaria Report 2019. Available online: https://www.who.int/publicationsdetail/world-malaria-report-2019 (accessed on 16 December 2019).

36. Aguzie, I.O.N.; Ivoke, N.; Onyishi, G.C.; Okoye, I.C. Antenatal Practices Ineffective at Prevention of Plasmodium falciparum Malaria during Pregnancy in a Sub-Saharan Africa Region, Nigeria. Trop. Med. Infect. Dis. 2017, 2, 15. [CrossRef]

37. Ouedraogo, S.; Bodeau-Livinec, F.; Briand, V.; Huynh, B.T.; Koura, G.K.; Accrombessi, M.M.; Fievet, N.; Massougbodji, A.; Deloron, P.; Cot, M. Malaria and gravidity interact to modify maternal haemoglobin concentrations during pregnancy. Malar. J. 2012, 11, 348. [CrossRef]

38. Anchang-Kimbi, J.K.; Nkweti, V.N.; Ntonifor, H.N.; Apinjoh, T.O.; Tata, R.B.; Chi, H.F.; Achidi, E.A. Plasmodium falciparum parasitaemia and malaria among pregnant women at first clinic visit in the mount Cameroon Area. BMC Infect. Dis. 2015, 15, 10. [CrossRef]

39. Di Renzo, G.C.; Spano, F.; Giardina, I.; Brillo, E.; Clerici, G.; Roura, L.C. Iron deficiency anemia in pregnancy. Womens Health (Lond.) 2015, 11, 891-900. [CrossRef] 
40. Agan, T.; Ekabua, J.; Udoh, A.; Ekanem, E.; Efiok, E.; Mgbekem, M. Prevalence of anemia in women with asymptomatic malaria parasitemia at first antenatal care visit at the University of Calabar Teaching Hospital, Calabar, Nigeria. Int. J. Womens Health 2010, 2, 229-233. [CrossRef]

41. Kefiyalew, F.; Zemene, E.; Asres, Y.; Gedefaw, L. Anemia among pregnant women in Southeast Ethiopia: Prevalence, severity and associated risk factors. BMC Res. Notes 2014, 7, 771. [CrossRef]

42. Hinderaker, S.G.; Olsen, B.E.; Bergsjo, P.; Lie, R.T.; Gasheka, P.; Kvale, G. Anemia in pregnancy in the highlands of Tanzania. Acta Obs. Gynecol. Scand. 2001, 80, 18-26. [CrossRef]

43. Nair, M.; Knight, M.; Robinson, S.; Nelson-Piercy, C.; Stanworth, S.J.; Churchill, D. Pathways of association between maternal haemoglobin and stillbirth: Path-analysis of maternity data from two hospitals in England. BMJ Open 2018, 8, e020149. [CrossRef]

44. Sukrat, B.; Wilasrusmee, C.; Siribumrungwong, B.; McEvoy, M.; Okascharoen, C.; Attia, J.; Thakkinstian, A. Hemoglobin concentration and pregnancy outcomes: A systematic review and meta-analysis. Biomed. Res. Int. 2013, 2013, 769057. [CrossRef]

45. Vural, T.; Toz, E.; Ozcan, A.; Biler, A.; Ileri, A.; Inan, A.H. Can anemia predict perinatal outcomes in different stages of pregnancy? Pak. J. Med. Sci. 2016, 32, 1354-1359. [CrossRef]

46. Berglund, S.K.; Torres-Espínola, F.J.; García-Valdés, L.; Segura, M.T.; Martínez-Zaldívar, C.; Padilla, C.; Rueda, R.; Pérez García, M.; McArdle, H.J.; Campoy, C. The impacts of maternal iron deficiency and being overweight during pregnancy on neurodevelopment of the offspring. Br. J. Nutr. 2017, 118, 533-540. [CrossRef]

47. Beard, J.L. Why iron deficiency is important in infant development. J. Nutr. 2008, 138, 2534-2536. [CrossRef]

48. Georgieff, M.K. The role of iron in neurodevelopment: Fetal iron deficiency and the developing hippocampus. Biochem. Soc. Trans. 2008, 36, 1267-1271. [CrossRef]

(C) 2020 by the authors. Licensee MDPI, Basel, Switzerland. This article is an open access article distributed under the terms and conditions of the Creative Commons Attribution (CC BY) license (http://creativecommons.org/licenses/by/4.0/). 